\title{
Essais cliniques et e-santé : impact des nouvelles technologies $d$ 'informations appliquées aux essais cliniques (y compris pour les données sources - dossier médical) et à la recherche sur la personne et le médicament
} \author{
Ronde $n^{\circ} 2$ de Giens XXV* \\ 1 Laboratoires MSD-Chibret, Paris, France \\ 2 Délégation Régionale à la Recherche Clinique et à l'Innovation, AP-HM, Marseille, France \\ 3 Agence Française de Sécurité Sanitaire des Produits de Santé, Saint-Denis, France \\ 4 Unité d’Épidémiologie des Maladies Émergentes, Institut Pasteur, Paris, France
}

Jehan-Michel Béhier ${ }^{1}$, Jean-Charles Reynier ${ }^{2}$, Pierre-Henri Bertoye ${ }^{3}$, Muriel Vray et les participants à la Table

Texte reçu le 4 mars 2010 ; accepté le 29 mars 2010

Mots clés :

e-santé ;

e-CRF ;

e-diary ;

essais cliniques

\begin{abstract}
Résumé - Les nouvelles technologies ont pris depuis quelques années une place importante dans notre environnement quotidien, qu'il soit professionnel ou personnel. Le monde de la santé n'a pas échappé à cette mutation progressive, avec l'arrivée de l'informatique jusqu'au chevet du malade. La recherche clinique a aussi trouvé un intérêt grandissant à tous ces nouveaux outils mis à la disposition aussi bien du médecin investigateur, du patient, que des services spécialisés qui contribuent au diagnostic et au suivi des patients, et aussi des différents métiers de la recherche clinique. Si l'usage des nouvelles technologies semble apporter des facilités, telles que la centralisation des informations ou la simplification des échanges de données entre différents intervenants, il n'en reste pas moins qu'il s'agit de données à caractère personnel, dont il faut assurer la fiabilité, la confidentialité et la sécurité, qu'elles soient utilisées dans le cadres des soins courants ou de celui de la recherche clinique académique ou industrielle.

L'objectif de la table ronde était d'évaluer l'impact des nouvelles technologies d'informations appliquées aux essais cliniques (y compris pour les données sources - dossier médical) et à la recherche sur la personne et le médicament. Dans un premier temps, un état des lieux du développement de ces nouvelles technologies dans l'environnement de la santé a été réalisé. La recherche des bénéfices attendus pour émettre des recommandations de bon usage ou des précautions d'emploi lors de leur utilisation dans le cadre des essais cliniques a été effectuée dans un second temps. Enfin, l'analyse de l'impact de ces nouvelles technologies tant du côté de l'investigateur, que de celui du promoteur, qu'il soit académique ou industriel a été estimée.
\end{abstract}

\section{Du dossier source à la base de données : état des lieux et pistes de réflexion}

1. 1. Données sources et dossiers patients

Le dossier médical du patient est un élément clé pour la recherche clinique. En effet, le dossier du patient retrace toute l'histoire médicale du patient que l'on devra documenter, que ce soit

\footnotetext{
* Pour la liste des participants, voir en fin d'article.
}

avant sa participation à un essai, pendant et au décours de l'essai. Les documents de ce dossier médical sont appelés dans le cadre de la recherche clinique «document sources », ${ }^{[1]}$ sur lesquels figurent des « données sources ». [2]

Autrefois le dossier médical du patient était un dossier papier, rassemblant les données cliniques et l'ensemble des résultats des examens paracliniques. Il permettait d'identifier les différents intervenants médicaux dans la vie du patient, au travers 
des courriers, des notes prises en consultation, et des résultats d'examens. Certains dossiers étaient incomplets voire difficilement interprétables tant l'histoire médicale du patient était complexe, d'autres étaient parfois réduits à leur plus stricte expression ou perdus au sein des archives, ou des services.

Aujourd'hui, grâce aux nouvelles technologies, le dossier médical d'un patient peut devenir un fichier (ou un ensemble de fichiers) informatique (Dossier Patient Informatisé ou DPI), centralisant toutes les informations médicales qu'il s'agisse de la description clinique ou des résultats des différents examens paracliniques. L'avantage indéniable de ce nouveau format est de permettre un partage plus aisé de ces informations d'un médecin à un autre, évitant ainsi l'interprétation de données illisibles et parfois la répétition d'examens. Son archivage est simplifié, et il est désormais plus facilement et plus rapidement consultable en raison de sa disponibilité immédiate.

Cependant pour que le partage des informations soit efficace tant pour le patient et les utilisateurs (médecins, infirmières, spécialistes, ... ) que sous l'angle technique, il est essentiel que ces informations respectent une certaine structuration homogène, des conventions de nommage, en bref tout un ensemble de règles et de pratiques adoptées par tous : une démarche de standardisation (comme par exemple HL7 aux États-Unis) ${ }^{[3]}$ permettant une interopérabilité entre les systèmes d'information concernés.

Par ailleurs, il est primordial, tant pour le patient, que pour les personnes amenées à consulter ces informations (personnels de santé, ou personnels habilités à suivre des essais cliniques), de pouvoir s'assurer de la véracité et de la traçabilité des informations médicales contenues dans ce dossier informatique, ainsi que du respect de l'intégrité et de la confidentialité des données.

Dans le cadre d'un essai clinique, le recueil des données patients s'effectue à partir des données du dossier médical du patient qui sont retranscrites dans le cahier d'observation ou du case report form $(C R F)$. Avec le dossier médical «papier» conventionnel, le recueil des données pertinentes pour l'essai clinique se faisait notamment par un report des données dans le CRF. Un contrôle était effectué par l'Attaché de Recherche Clinique (moniteur ou ARC) qui comparait les données du dossier médical avec celles reportées dans le cahier d'observation, permettant ainsi de vérifier la cohérence, et de faire corriger les données erronées ou manquantes par le médecin investigateur.

Avec le dossier médical électronique, le processus de vérification des données reste malheureusement pour l'instant identique.

En effet, aujourd'hui, la traçabilité et la fiabilité des informations médicales d'un dossier «patient» électronique ne sont pas totales en raison d'un manque de standardisation du processus d'enregistrement des données, de la traçabilité des modifications de ces données, donc d'une interprétation de ces dernières, et de l'absence, en général, de garanties sur leur stabilité et leur intégrité au sein du système informatique qui les gère (audit trail). Cela a deux conséquences potentielles :

- Une impression des données sources nécessaires à la conduite du monitoring de l'essai clinique, impression qui sera datée et signée par l'investigateur.

- L'obligation pour l'investigateur (ou la personne déléguée par l'investigateur) de reporter les données du patient dans le CRF, qu'il soit électronique ou papier, et d'assurer un contrôle de ces données reportées par le représentant du promoteur (ARC).

En résumé, si le principe du dossier informatique est aujourd'hui bien implanté, que ce soit dans les services hospitaliers ou les cabinets libéraux (spécialistes ou médecins généralistes), les limites de son utilisation en tant que dossier source en recherche clinique tiennent principalement à un manque de standardisation et à un niveau de sécurité insuffisant pour assurer la fiabilité et la pérennité des données.

Aujourd'hui, quatre critères sont nécessaires pour qu'un dossier patient informatisé puisse être considéré comme le document source :

- Adoption de standards dans l'appellation des données et dans leur structuration (structuration informatique mais aussi structuration sémantique, par exemple en ayant recours à MeDdRA).

- Mise en place d'accès et de droits individualisés en écriture ou en lecture, selon les profils des intervenants, sur le dossier informatique (médecin, infirmière, technicien essai clinique, secrétaire...).

- Existence d'un audit trail, ou piste d'audit : le système informatique devra enregistrer et identifier toutes les modifications effectuées et les personnes qui les ont faites, les raisons de ces modifications, ainsi que la date à laquelle elles ont été réalisées.

- Existence de sauvegardes régulières et archivage fiable des données.

Sous ces conditions et grâce aux nouvelles technologies, la récupération des données d'un patient et leur retranscription dans le CRF électronique pourraient s'effectuer de manière électronique et sans faire appel à des supports intermédiaires papiers. Ce transfert de données doit se faire avec la mise en place de protocoles validés de transfert de données spécifiques depuis le dossier médical du patient vers le CRF électronique.

\subsection{Dossiers patients et base de données patients}

Un autre avantage majeur du remplacement du dossier médical papier par un dossier électronique est la possibilité pour le 
médecin intéressé par la conduite d'une recherche clinique d'avoir accès à une base de données constituée de l'ensemble des données cliniques et paracliniques de ses patients.

Sous réserve d'une standardisation minimale (par exemple, adoption des règles issues de HL7/CDISC et généralisation de l'emploi de dictionnaires comme MeDdRA) dans la façon de saisir les informations des patients vus en consultation ou en hospitalisation, le médecin ou un membre de son équipe pourra, à tout moment et relativement simplement, faire des requêtes au sein de cette base pour identifier des patients éligibles à une recherche spécifique, qu'elle soit de nature épidémiologique ou thérapeutique.

Cependant, à ce jour, il est constaté qu'il perdure un manque de standardisation, d'homogénéisation, de compatibilité et d'interopérabilité entre les différents systèmes de collectes des données des patients qui ont été mis en place auprès des professionnels de santé, que ce soit en ville ou à l'hôpital.

De plus, nous observons une redondance de bases, en particulier au sein des hôpitaux (DPI, PMSI, autres bases de données provenant des laboratoires d'analyse ou des services d'imagerie, ... ) sans réelle possibilité d'interface entre elles.

Sous réserve du respect des exigences légales et en particulier des formalités préalables devant la Commission Nationale Informatique et Libertés (CNIL) et de l'information des personnes concernées, les progrès techniques devraient permettre la fusion de ces différentes bases en limitant la redondance des informations. Cette interconnexion entre bases permettrait ainsi une exhaustivité des données mises à disposition des équipes de recherche clinique afin d'être plus précis dans la recherche de profils patients éligibles pour un projet de recherche donné (file active de patients).

Un développement de bases standardisées (dotées d'une véritable architecture informatique d'une base de données) munies de système de traçabilité (piste d'audit ou audit trail) des entrées et de toutes modifications des données (cliniques et paracliniques) est nécessaire. Pour parvenir à cet objectif ambitieux, il faut définir en amont, avec les utilisateurs, les besoins concernant l'outil (utilisation, type de requête, ...), la liste exhaustive des données à saisir ou les informations provenant d'autres services à intégrer (biologie, imagerie, ....) et sur cette base adopter ensemble des standards soit publiques, soit spécifiques si les standards publiques ne couvrent pas la spécificité des données recherchées. Le développement de ce type de base passe par la mise en place des spécifications fonctionnelles, par l'analyse des risques et la validation technique du développement informatique. Un panel de tests réalisés par différents types d'utilisateurs viendra compléter les tests de la base avant la mise en production de l'outil.

La technologie et les outils informatiques permettent assez simplement de répondre aux besoins des utilisateurs, mais il est important de noter que le manque de moyens, tant humains que logistiques, limite la mise en place et la gestion correcte de ces nouveaux outils.

Un autre élément critique (et qui d'ailleurs est une conséquence de l'analyse de risque) est la formation des utilisateurs et l'assurance que cette formation est bien comprise.

\subsection{Le cahier d'observation électronique (e-CRF) et la base de données de l'étude}

La mise en place du cahier d'observation électronique (eCRF) dans la conduite des essais cliniques a changé de façon substantielle la coopération entre les différents partenaires de la recherche clinique.

Tout d'abord sur le plan humain, le dialogue entre le moniteur ou l'ARC et le médecin investigateur, autrefois centré exclusivement autour du patient lors du travail de contrôle de cohérence fait sur site par l'ARC, est maintenant remplacé par une discussion sur les modalités pratiques de remplissage du cahier d'observation et de gestion des questions ou demandes de clarification (queries, voir ci-dessous) transmises électroniquement, ainsi que sur les difficultés techniques rencontrées. De plus, l'eCRF est à l'origine de nouvelles fonctions de personnes chargées de gérer, sous la responsabilité de l'investigateur, les activités de saisie des données dans le cahier d'observation. Le contrôle de la cohérence des données est maintenant basé sur l'exécution de queries directement dans l'e-CRF, alertant sur la saisie de données erronées, incohérentes ou manquantes, au moyen de différents signaux d'alerte. Ces queries sont générées, en partie, automatiquement (par exemple sur des incohérences simples comme l'âge ou le poids) ou plus classiquement comme aujourd'hui suite à une revue des données.

Il est à noter que ce mécanisme de queries est associé à un $a u$ dit trail donc à une traçabilité rigoureuse des modifications éventuelles des données saisies dans l'e-CRF.

Par ailleurs, le développement et l'utilisation de l'e-CRF pour les essais cliniques sont une avancée positive évidente pour faciliter la conduite d'un essai clinique, notamment en raison de la mise à disposition rapide de l'ensemble des données cliniques en temps réel.

Pour l'investigateur, les bénéfices immédiats sont, outre l'encombrement moindre (pas de classeur patient parfois volumineux à garder tout au long de l'étude), une gestion simplifiée des données par la présence de contrôles automatiques, réduisant ainsi certaines requêtes pour clarifier des informations (queries ou DNF), un accès facilité aux données, une sauvegarde et un archivage simplifiés.

Pour le promoteur de la recherche, le bénéfice premier est un gain de temps dans la gestion des données (absence de double 
saisie et élimination des difficultés de relecture d'écriture manuelle parfois illisible, collecte et accès aux données en temps réel, codage des données en ligne, traçabilité des corrections de données, chargement direct des données biologiques ou autres examens paracliniques centralisés, ... ). C'est, pour le promoteur, la possibilité d'avoir en temps réel une liste des évènements indésirables en cours d'essais et ainsi de pouvoir répondre immédiatement aux Autorités de Santé, conformément aux exigences légales et réglementaires définies par le Code de la Santé Publique. ${ }^{[4]}$

Si l'intérêt de l'e-CRF ne fait aucun doute pour les études multicentriques, qu'elles soient nationales ou internationales, notamment pour des aspects de facilité opérationnelle de gestion de données, de simplification et d'optimisation des monitoring par accès direct et en temps réel aux données des patients nécessaires au contrôle de la qualité par les ARCs, le bénéfice attendu peut sembler moins crucial pour les études mono-centriques.

En effet, pour les études mono-centriques, l'usage de l'eCRF peut sembler moins évident, notamment dans une première approche si l'on compare par exemple la simplicité d'élaboration d'un cahier d'observation papier par rapport à la construction d'une interface informatique qui nécessite des compétences techniques particulières. Néanmoins, lorsque l'on confronte l'ensemble de la chaîne qui va être en charge de la gestion des données, de leur analyse et de leur utilisation, le cahier d'observation électronique et la traçabilité des informations devraient être les garants d'une meilleure qualité, comparativement au cahier papier et à la feuille de calcul sous Excel. Les bénéfices attendus pourront être au-delà du projet de recherche lui-même en permettant des agrégations de données plus faciles entre études (en respectant les déclarations réglementaires préalables). La réduction ou la disparition du support papier facilitera l'archivage et le stockage tout en dynamisant la transmission des données.

Par ailleurs, la fiabilité, la traçabilité et l'authentification des données sont assurées par l'outil informatique au travers de procédures opératoires standardisées et d'outils, que ce soit par des accès nominatifs, la signature électronique mais aussi l'audit trail qui permet de suivre les différents intervenants et les différentes interventions dans la base de données. De même, l'e-CRF et la base de données ainsi constituée feront l'objet de maintenances et de sauvegardes régulières.

Ainsi, in fine, un système informatisé est une meilleure garantie de la qualité des données donc une meilleure garantie pour le patient dans sa prise en charge.

Bien entendu, l'utilisation de l'e-CRF nécessite des ressources humaines et matérielles, et des compétences techniques additionnelles. Pour les recherches académiques ou institutionnelles ne disposant pas d'importants moyens financiers et techniques, il serait important de soutenir les initiatives visant à proposer des bases simples d'utilisation, avec des «process » d'utilisation validés (audit trail, maintenance, archivage) et des masques de saisie standardisés, facilement adaptables au type de recherche. La création de la future base de données d'une étude doit également être associée à une réflexion portant sur les données à recueillir (intérêts et utilisation), ainsi que sur les contrôles de cohérence à effectuer. Une coordination doit être mise en place dès la conception de l'e-CRF entre le médecin investigateur, la personne en charge de compléter l'e-CRF et les informaticiens.

Aujourd'hui, alors que l'e-CRF est devenu pratique courante dans les essais multicentriques, on constate que les utilisateurs, investigateurs, moniteurs ou attachés de recherche clinique, sont parfois réticents à l'utilisation de ces nouveaux outils. Pourtant, il est également constaté que les bénéfices attendus sont réels notamment en termes de disponibilité, de suivi, de corrections des données et d'échanges d'informations.

Pour vaincre les réticences des acteurs de la recherche clinique, plusieurs pistes de réflexion peuvent être proposées, qui reposent toutes sur la formalisation des processus, des méthodes et des outils de travail partagés par l'ensemble des acteurs impliqués :

- Mettre à disposition des utilisateurs une formation présentant à la fois les intérêts et avantages de ces nouveaux outils, mais aussi leur rappelant les obligations légales et leurs responsabilités, ainsi que la façon dont ils doivent les utiliser (confidentialité, respect des codes et mot de passe personnels, mention CNIL à l'ouverture des outils, ... ). Cette formation doit aussi présenter les modalités de remplissage des différents éléments de l'e-CRF.

- Reconnaître les nouveaux métiers impliqués dans la recherche clinique, ainsi que leurs compétences, et être en mesure de documenter et de tracer les niveaux de délégation et d'intervention sur ces bases de données. Harmoniser les pratiques.

- Développer une reconnaissance des e-training, e-learning et certifications conçus pour un même outil.

\subsection{Le carnet patient électronique (e-diary)}

Le carnet patient sous format papier au cours d'un essai clinique est très souvent difficile à exploiter, en raison des incertitudes du report des données par le patient, notamment au cours du temps (traçabilité, fiabilité des horaires et de l'information recueillie), de l'impossibilité d'interroger le patient a posteriori au moment de la saisie des données illisibles ou incohérentes, alors que les informations rapportées directement par le patient pourraient s'avérer très utiles.

Le carnet patient électronique est de plus en plus souvent utilisé au cours des essais cliniques. Il a pour objectif d'améliorer la qualité du recueil de l'information dès le début et tout au long 
de l'essai, par l'utilisation d'alarmes programmées (rappels pour compléter les questionnaires) et avec l'aide éducative de l'investigateur auprès du patient.

De plus, l'investigateur assure, dans une certaine mesure, la véracité de l'information (code d'accès pour le patient, chargement de l'information directement dans la base de données de l'essai). Dans certains cas, il peut également suivre en temps réel les informations saisies par son patient sur un site internet, et éventuellement le contacter en cas d'oubli.

L'usage du carnet patient peut toutefois avoir des limites : par exemple, la capacité du patient à comprendre les explications qui lui sont fournies par l'investigateur ou celles en relation avec sa pathologie.

La mise en place de ce type d'outil nécessite que le personnel en charge de la recherche soit lui-même formé à son utilisation et sache expliquer au patient les modalités pratiques de ce carnet électronique de surveillance. Il faut aussi que l'investigateur puisse avoir les moyens d'évaluer l'observance du patient, surtout lorsque le critère principal d'évaluation de l'essai repose sur ces données.

Là encore, ceci passe par un effort réel visant à expliquer à chaque acteur comment le carnet patient s'insère dans la chaine clinique, ce qu'il apporte par exemple à chaque patient dans sa prise en charge, donc à un effort inhabituel de formalisation du processus global.

\subsection{Transfert de données électroniques}

Deux situations de transfert de données ou de documents ont fait l'objet d'une discussion.

\subsubsection{Transfert de données entre le Promoteur et les Autorités de Santé}

Dans le cadre de la conduite d'essais cliniques en France, le transfert des données, qu'il s'agisse de transfert de documents ou de données, entre le promoteur et les autorités doit être discuté. Le constat unanime et favorable envers l'expérience acquise avec l'Agence Française de Sécurité Sanitaire des Produits de Santé (Afssaps) lors des dépôts réglementaires pour le transfert de données ou de documents conduit à recommander une harmonisation des pratiques de transmission tout particulièrement entre les deux instances réglementaires que sont les Comités de Protection des Personnes et l'Afssaps. La Table Ronde recommande donc fortement le développement du format d'échange commun, garantissant la confidentialité des données, de type Eudralink dans un premier temps, suivi d'une standardisation des données et informations échangées dans un deuxième temps, par exemple en s'inspirant des recommandations HL7/CDISC. ${ }^{[3,5]}$

\subsubsection{Transfert de données ou de base de données entre investigateur, promoteur et prestataires de service}

Le transfert de données ou de bases de données entre le promoteur, l'investigateur et les prestataires de service peut sembler une évidence en soi, tant l'échange d'informations se fait de façon courante et sans réelle limitation, notamment technique. Le respect de la législation au titre de la mise en œuvre d'un traitement de données à caractère personnel implique l'identification précise des catégories de destinataires de données au sein du dossier déposé devant la CNIL et le Comité Consultatif sur le Traitement de l'Information en Matière de Recherche dans le domaine de la Santé (CCTIRS) [ou, le cas échéant si elle est applicable à l'étude concernée, conformément à la Méthodologie de référence MR 001], la mise en place de mesures de sécurité permettant d'assurer la confidentialité des, mais également le respect des dispositifs légaux d'encadrement du transfert des données intra-européen (principe de libre circulation des données) et extra-européen (SAFE HARBOR, conventions de flux transfrontaliers ou encore binding corporate rules) : il est important de rappeler que ce transfert se fait sous la responsabilité du promoteur en toutes hypothèses. Les données transférées ne doivent pas servir une autre finalité que celle de la recherche (finalité déclarée au sein de la demande d'autorisation adressée à la CNIL ou le cas échéant, si elle est applicable à l'étude concernée, conformément à la méthodologie de référence MR 001).

Il est important de suivre le maintien de l'intégrité des données à chaque échange ainsi que la sécurisation globale du système de transmission. Une forte recommandation est le développement d'un format d'échange standardisé et sécurisé de type CDISC et de son adoption comme format réglementaire au sein de l'Union Européenne.

Associé à cette recommandation, il importe dans les contrats unissant les différents acteurs de s'assurer que les protocoles de format, stockage et échange des données sont clairement établis et auditables.

Concernant les bases de données des essais cliniques, en l'état, il n'y a pas de recommandation complémentaire à formuler par rapport à l'existant. Cependant, cet accès facilité aux données qui permet une prise de décision plus rapide, notamment dans le cadre des data and safety monitoring board (DSMB), doit se faire en conformité avec les Bonnes Pratiques Cliniques. En effet, le risque d'interprétation de résultats (analyse sauvage, cachée) à partir de données agrégées ou non doit être totalement contrôlé, et l'étanchéité entre les données «masquées» et les fichiers de décodage du tirage au sort doit être assurée et doit pouvoir être prouvée.

De même, il est rappelé que les aspects éthiques ne doivent pas être abordés différemment qu'il s'agisse d'une compilation 
de données sous format électronique ou sous format papier, avec la nécessité dans les deux cas d'afficher les mentions légales de confidentialité et d'information, et lorsque la loi l'exige, de recueillir le consentement des personnes concernées.

Un effort concernant l'anonymisation et le codage des documents para-cliniques (CD d'imagerie, données biologiques, ...) est demandé en incitant le développement de logiciels adaptés aux impératifs du soin courant et de la recherche.

\section{Recommandations et points clé}

Les nouvelles technologies d'information appliquées aux essais cliniques apportent des avantages incontestables dans la gestion des projets de recherche, tant par leur facilité d'utilisation, que dans la mise à disposition plus rapide des résultats et donc in fine dans la prise en charge des patients. Néanmoins, il est important pour les concepteurs, comme pour les utilisateurs, de bien prendre en compte les règles d'utilisation de ces nouvelles technologies. En effet, que ce soit pour la recherche clinique, ou la collecte de données de santé d'un patient, il est absolument nécessaire d'assurer la compréhension (standards), la fiabilité, la traçabilité et la confidentialité des données et ce dans un contexte de conformité légale et de sécurité juridique.

\subsection{Dossier source / dossier médical de patients}

- Standardisation de la codification et de la structuration des données/informations.

- Développement et utilisation de logiciels et de bases de données sécurisées disposant d'un audit trail (piste d'audit).

- Droits d'accès individualisés et mot de passe personnel.

- Formation des utilisateurs et documentation de la formation.

- Sauvegarde systématique et archivage fiable.

\section{2. e-CRF - e-Diary}

- Formalisation des processus et des droits et devoirs de l'ensemble des acteurs cela inclus à la fois la responsabilité sur la création et l'usage des données, mais aussi sur la façon dont la création et l'usage contribuent aux enjeux finaux de la recherche et de la prise en charge des patients.

- Responsabilisation des utilisateurs (accès personnel, rôles et délégations documentées).

- Reconnaissance des e-trainings faits pour un même outil informatique.

- Rappel des obligations en matière de protection des données : mention CNIL à l'ouverture des outils, information sur les droits (accès, ....) et les devoirs (confidentialité,...).

\subsection{Transfert des données}

- Préconisation d'un espace d'échange commun sécurisé, type Eudralink pour tous les échanges avec les autorités (Afssaps et CPP).

- Développement d'un format d'échange standardisé et sécurisé de type CDISC et de son adoption comme format réglementaire au sein de l'Union Européenne, devant sécuriser les données tout au long de la vie de l'étude clinique afin d'éviter toute analyse «sauvage » ou cachée.

\subsection{Base de données des études cliniques}

- Pas d'impact des nouvelles technologies d'information qui mettent à disposition les données en temps réel.

\subsection{Aspects éthiques}

- Même cadre légal que pour le papier - Loi informatique, Fichiers et Libertés.

- Nécessité d'afficher les mentions légales de confidentialité et d'information :

- Au patient (avec le consentement si il est légalement requis), comme à tout intervenant dans la recherche.

- À adapter selon le support.

- Recommandation : inciter au développement d'outils permettant l'anonymisation des données électroniques (CD imagerie, ou données biologiques).

Plusieurs de ces points font actuellement l'objet de groupes de travail et devraient conduire prochainement à la publication de recommandations tant sur la gestion des données que sur leur traçabilité et leur sécurisation.

\subsection{Un sujet complémentaire non abordé}

Tous les éléments décrits ci-dessus concourent aussi à la constitution de la documentation associée à la recherche clinique quelle que soit sa nature.

Cette documentation, aujourd'hui majoritairement électronique, est utilisée de plusieurs manières :

- Pour être transmise aux autorités en amont, pendant et en aval de la recherche clinique.

- Pour argumenter une mise sur le marché ou un dossier de transparence.

- Pour suivre les effets indésirables et apporter au patient d'abord aux autorités ensuite la vigilance nécessaire. 
- Pour constituer un référentiel : le Trial Master File.

Il serait intéressant lors d'un prochain séminaire de Giens d'aborder la jonction entre le recueil de données et la documentation, ainsi que la gestion de cette dernière.

\section{Conclusions}

Les nouvelles technologies ont pris une place très importante dans le monde de la santé, comme dans celui des essais cliniques pour la recherche sur la personne et le médicament. Leur usage a bouleversé certaines habitudes et pratiques. Ces outils sont parfois très organisés et structurés.

La Table Ronde recommande très fortement de communiquer sur les enjeux de l'e-santé auprès des acteurs de santé, rappelant les bénéfices attendus de ces nouvelles technologies d'information, non seulement dans le cadre de la recherche clinique (qu'elle soit institutionnelle, académique ou industrielle), mais aussi du point de vue de la responsabilité médicale : standardisation et traçabilité des données recueillies afin d'en optimiser la qualité et la fiabilité, et d'en faciliter l'accès. Ceci permettra ainsi aux équipes de recherche clinique d'être des acteurs compétitifs et reconnus dans leur capacité à identifier des patients éligibles pour une recherche clinique, favorisant ainsi la prédictibilité de leurs engagements en termes de recrutement. C'est aussi un accès plus rapide aux données pour une prise de décision plus rapide, notamment dans le cadre des DSMB. Cependant, cette facilité d'accès aux données doit impérativement se faire en conformité avec les Bonnes Pratiques Cliniques et la législation sur le traitement des données personnelles. L'enjeu des nouvelles technologies en recherche clinique est incontournable, mais ne pourra être performant et opérationnel que s'il s'accompagne de moyens humains et logistiques.
Participants. Béatrice Barraud (Inserm, Paris), JehanMichel Béhier (Laboratoire Merck Sharp Dohme-Chibret), PierreHenri Bertoye (Afssaps, Saint-Denis), Nathalie Beslay (Avocate, Paris), Julie Boussuge (CHU, Bordeaux), Loïc Chartier (Institut Pasteur, Paris), Erick Gaussens (Laboratoire Product Life), Marta Gersberg (Laboratoire Sanofi-Aventis), Eric Leutenegger (Laboratoire ABR Pharma), Monique Malchiodi (Laboratoire Roche), Dominique Menuet (Laboratoire Pfizer), Noureddine Namir (Laboratoire GlaxoSmithKline), Jean-Charles Reynier (AP-HM, Marseille), François Rivaille (Laboratoire Servier), Stéphanie Sallman (Afssaps, Saint-Denis), Marie Sebille (Laboratoire Sanofi-Aventis), Gilles Sonou (Peter Holmes), Muriel Vray (Inserm/ Institut Pasteur, Paris).

\section{Références}

1. Décision du 24 novembre 2006 fixant les règles de bonnes pratiques cliniques pour les recherches biomédicales portant sur les médicaments à usage humain. Glossaire, définition 1.17. http: //www . legi france.gouv. fr

2. Décision du 24 novembre 2006 fixant les règles de bonnes pratiques cliniques pour les recherches biomédicales portant sur les médicaments à usage humain. Glossaire, définition 1.18. http://www.legifrance.gouv.fr

3. www.hl7.org

4. Décret $\mathrm{n}^{\circ} 2006-477$ du 26 avril 2006 modifiant le chapitre 1er du titre II du livre Ier de la première partie du Code de la Santé Publique relatif aux recherches biomédicales. Art. R.1123-44. http://www. legifrance.gouv. fr

5. http: //www. cdisc.org

Correspondance et offprints : Jean-Charles Reynier, Délégation Régionale à la Recherche Clinique et à l'Innovation, AP-HM, CHRU de Marseille, 13354 Marseille Cedex 05, France.

E-mail : JCREYNIER@ap-hm.fr 